\title{
TIN OXIDE MICROSENSORS ON THIN SILICON MEMBRANES
}

\author{
Shih-Chia Chang \\ David B. Hicks \\ Electronics Department \\ General Motors Research Laboratories \\ Warren, Michigan 48090-9056
}

\section{Abstract}

Tin oxide based microsensors with integrated polysilicon heaters were fabricated on thin silicon membranes ( $-2 \mu \mathrm{m}$ thick) generated by anisotropic wet chemical etching using ethylene diamine-pyrocatechol (EDP) as the etchant. Good thermal isolation and lower power consumption were achieved. The sensing elements, tin oxide thin films, were prepared either by sputter-deposition or by metallo-organic deposition (MOD). The completed microsensors showed good response to alcohol vapor and good stability of the integrated polysilicon heaters.

\section{Introduction}

Semiconductor gas sensors generally have to be operated at elevated temperatures $\left(150^{\circ} \mathrm{C}-700^{\circ} \mathrm{C}\right)$ to attain the speed and magnitude of response required for practical applications [1-3]. Consequently, for the development of portable gas sensors or integrated sensing systems with on-chip control/logic circuits, effective thermal isolation for the active sensing area becomes imperative in order to keep the power consumption low and to protect adjacent circuitry from extreme temperature generated by the sensor heater. One way of attaining low power consumption and good thermal isolation is by fabricating a sensing element with an integrated heater in thin film form on a suspended thin membrane. By doing this, both the heating mass and heat transport path are greatly reduced. Silicon membranes can be readily generated by an anisotropic wet chemical etching technique using either potassium hydroxide (KOH) or ethylene diaminepyrocatechol (EDP) as the etchant $[4,5]$. Such a technique has been used to fabricate piezoresistive or piezocapacitive pressure transducers $[6,7]$. When using EDP, precise control of the membrane thickness can be achieved by using a heavily doped boron layer as the etch-stop.

The main purpose of this work is twofold: a) to fabricate tin oxide-based microsensors on micromachined thin membranes to attain good thermal isolation, and b) to evaluate sputter-deposited and metallo-organic deposited tin oxide thin films.

This paper describes the detailed processing procedures used to fabricate these microsensors and also presents the various sensor responses to reducing gaseous species (propylene, vapors of alcohol, ethyl ether, methoxyflurane and halothane).

\section{Experimental}

\section{Device Fabrication}

A five-mask process was used to fabricate tin oxide thin film microsensors on silicon membranes.
Most of the processing steps are similar to those used in our previous work [8]. The major additions and modifications adopted in the current fabrication process are described below:

a) The formation of a thin silicon membrane

A silicon membrane with a thickness of $-2 \mu \mathrm{m}$ was formed by anisotropic chemical etching using EDP. EDP has a fast etch rate $(-80 \mu \mathrm{m} / \mathrm{h})$ in the $\mathrm{Si}$ $\langle 100\rangle$ direction, a relatively slower etch rate $(<10 \mu \mathrm{m} / \mathrm{h})$ in the $\mathrm{Si}\langle 111\rangle$ direction, a very slow etch rate $(<20 \mathrm{~nm} / \mathrm{h})$ for $\mathrm{SiO}_{2}$, and a close-to-zero etch rate for heavily boron doped silicon. Thus, a heavily boron doped layer (doping concentration $\geq 5 \times 10^{19} \mathrm{~cm}^{-3}$ ) generated by ion implantation (dosage $-5 \times 10^{16} \mathrm{~cm}^{-2}$, implant voltage $-200 \mathrm{keV}$ ) was used as the etch-stop.

b) The formation of the sensing element

Tin oxide thin films were prepared either by sputter-deposition [3] or by metallo-organic deposition (MOD) [9]. For the sputter-deposited samples, the sensing element processing steps are the same as those used in our previous work. For the MOD technique, an ink was prepared by dissolving tin (II) 2-ethylhexanoate in xylene. The ink was spun onto a silicon wafer, fired to form a $100 \mathrm{~nm}$ to $200 \mathrm{~nm}$ tin oxide $\mathrm{film}$, and subsequently patterned either by reactive ion etching or by wet chemical etching.

c) The formation of metal interconnects

An aluminum (Al)/chromium ( $\mathrm{Cr}$ ) double layer with a thickness of $1 \mu \mathrm{m} / 50 \mathrm{~nm}$ was used to form metal interconnects for the tin oxide as well as the polysilicon heater. Chromium not only provides good ohmic contact for tin oxide, it also prevents electromigration of the interconnect metals at the heater contacts. Severe electromigration was observed if only Al was used as the contact metal, and would result in poor contact or premature contact failure. The processes used to form the MOD tin oxide metal interconnects had to be modified due to the high reactivity of the MOD films. The most commonly used process for metal interconnect fabrication consists of the following steps: a) Deposition of metal(s) on top of the material to be electroded, b) photolithographically defining the interconnect features into a photoresist layer, and c) transferring the defined feature into the underlying metal layer by wet chemical etching. However, when the above procedure is used, two problems are encountered:

(1) During wet chemical etching of $A l$, active hydrogen $\left(H^{*}\right)$ is produced at the metal/MOD tin 
oxide interface according to the following general equation:

$$
\mathrm{Al}+\mathrm{H}(\mathrm{X}) \longrightarrow \mathrm{Al}(\mathrm{X})+\mathrm{H}^{*}
$$

where $\mathrm{H}(\mathrm{X})$ is an etchant composed of $\mathrm{H}_{3} \mathrm{PO}_{4}, \mathrm{HNO}_{3}$, and $\mathrm{CH}_{3} \mathrm{COOH}$. The MOD tin oxide is readily' reduced to $\operatorname{tin}^{3}$ by $\mathrm{H}^{*}$,

$$
\mathrm{SnO}_{2}+4 \mathrm{H}^{*} \longrightarrow \mathrm{Sn}+2 \mathrm{H}_{2} \mathrm{O}
$$

and is removed by the etchant, resulting in a bad (or open) tin oxide contact.

(2) In photolithographic processes for the patterning of the metal interconnect feature, the weak chemical reaction between the hydroxide-based positive resist developer and $\mathrm{Al}$ produces active hydrogen

$\mathrm{Al}+3(\mathrm{OH}) \rightarrow \mathrm{Al}(\mathrm{OH}) \underset{3}{\rightarrow} \mathrm{AlO}_{3}^{-}+\mathrm{H}_{3} \mathrm{O}^{+}$

The reduction of $\mathrm{SnO}_{\mathrm{O}}$ by the active hydrogen as expressed by $\mathrm{Eq} .2$ at the metal/SnO interface degrades the metal contact of MOD tin oxide film.

Two approaches were used to overcome the MOD tin oxide/metal contact problems: a) by using a lift-off technique [10]; b) by using an inverse metallization technique. In the lift-off technique, the MOD tin oxide thin film sensing element was prepared as described in the previous section, an opposite contrast metal interconnect feature was defined in a positive photoresist layer and $\mathrm{Al} / \mathrm{Cr}$ was then deposited. The metal on top of the photoresist layer was subsequently lifted off by acetone. In this process, the copresence of positive photoresist developer, MOD tin oxide and metal was avoided, and wet chemical etching of the metal interconnect was eliminated. In the inverse metallization scheme, the $\mathrm{Al} / \mathrm{Cr}$ metal interconnect was fabricated prior to the deposition of the MOD film. The Al layer at the metal/SnO area was etched off, exposing the underlying $\mathrm{Cr}$ layer. The MOD tin oxide sensing element was then fabricated as described before. In both cases, the chemical reaction expressed in Eq. 2 was avoided and a reliable metal/MOD tin oxide contact was obtained. The schematic diagrams of the cross-sectional views of the inverse metallization structure and conventional metallization structure are shown in Fig. 1. A complete microsensor structure is shown schematically in Fig. 2 .

Figures $3 a$ and $3 b$ are the SEM pictures of a finished tin oxide microsensor on a $\mathrm{Si}$ membrane, showing the top and cross-sectional views, respectively. The dimensions of the sensing element (the sensing element was a sputterdeposited tin oxide, in this particular case) are $25 \mu \mathrm{m} \times 25 \mu \mathrm{m}$.

\section{Results and Discussion}

The electrical resistance of tin oxide film strongly depends on its stoichiometric composition and can be effected by fabrication processes. For instance, in the reactive ion etching (RIE) process, the sample was immersed in an intense plasma containing reactive reducing gaseous species such as chlorine radicals. A decrease in sample resistance was observed after the RIE process. (The sample resistance was restored after a proper heat treatment in air.) Oxygen plasma exposure, on the other hand, tends to increase the tin oxide resistance due to its strong oxidation strength. Hence, in fabricating tin oxide microsensors as well as other transition metal oxide-based sensors, the effects of microfabrication processes on the properties of the sensing materials (intrinsic sample conductance as well as gas sensitivity) should be carefully investigated. From our work on tin oxide microsensors, we found that although the sensor resistance can be affected by the RIE and oxygen plasma treatments, no discernible, detrimental effect on the gas sensitivity by such treatments was observed. In this work, the exposure of tin oxide films to an oxygen plasma was kept to a minimum or totally avoided.

The responses of the MOD tin oxide thin film microsensors to propylene and to vapors of ethyl alcohol, ethyl ether, methoxyflurane and halothane in air are shown in Fig. 4. The relative sensitivities of the microsensor to the organic vapors tested are, in decreasing order, ethyl alcohol $>$ ethyl ether > methoxyflurane > halothane. This is congruous with the relative chemical activeness of the respective molecular species. The alcohol vapor sensitivity of the current microsensor is about one third of that given by a similar sensor that has not been subjected to micromachining processes. This indicates that the protective black wax coating on top of the sensing device during the EDP cavity etching process has a detrimental effect on the alcohol sensitivity of the tin oxide sensor.

The integrated sensor heater was a boron doped polysilicon layer with a thickness of -5000 A. The sheet resistance of the heater versus temperature is shown in Fig. 5. In this experiment, a voltage of $-3.6 \mathrm{~V}$ and a current of $-40 \mathrm{~mA}$ were applied to the heater, which is equivalent to a power consumption of $\sim 0.15$ watt. From the $R(T)$ curve of Fig. 5, the sensor temperature was estimated to be $-250^{\circ} \mathrm{C}$.

\section{Summary}

Microsensors with tin oxide sensing elements and integrated polysilicon heaters were fabricated on thin silicon membranes generated by anisotropic wet chemical etching using ethylene diamine-pyrocatechol (EDP) as the etchant. The fabricated microsensors had good thermal isolation and low power consumption, and showed good response to organic vapors such as ethyl alcohol and ethyl ether.

\section{Acknowledgements}

We would like to thank M. Putty, J. Biafora and C. Puzio for their assistance in microfabrication, $R$. Laugal and A. Micheli for providing metallo-organic inks and $W$. Lange for taking the SEM pictures.

\section{References}

[ 1] S. R. Morrison, Sensors and Actuators, 2, 329 (1982).

[ 2] G. R. Heiland, Sensors and Actuators, 2, 343 (1982).

[ 3] S. C. Chang, IEEE Trans. Electron Devices, ED26, 1875 (1979).

[ 4] K. E. Petersen, Proceedings of IEEE, 70, 420 (1982). 
[ 5] N. F. Raley, Y. Sugiyama, and T. V. Duzer, J. Electrochem. Soc. 131, 161 (1984).

[ 6] S. K. Clark and K. D. Wise, IEEE Trans. Electron Devices, ED-26 1887, (1979).

[ 7] C. S. Sander, J. W. Knutti, J. D. Meindl, IEEE Trans. Electron Devices ED-17, 927, (1980).

[ 8] S. C. Chang and D. B. Hicks, GM Research Publication, GMR-4954, (1985).

[ 9] S. C. Chang and A. L. Micheli, GM Research Publication GMR-5212, (1985).

[10] M. Hatzakis, J. Electrochem. 116, 1033 (1969).

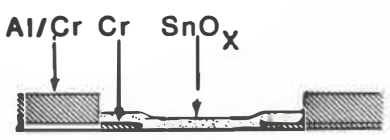

(a)

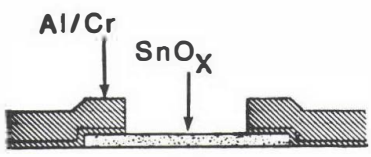

(b)

Fig. 1 Schematic diagrams of the cross-sectional view of the inverse metallization structure (a), and the conventional metallization structure (b).

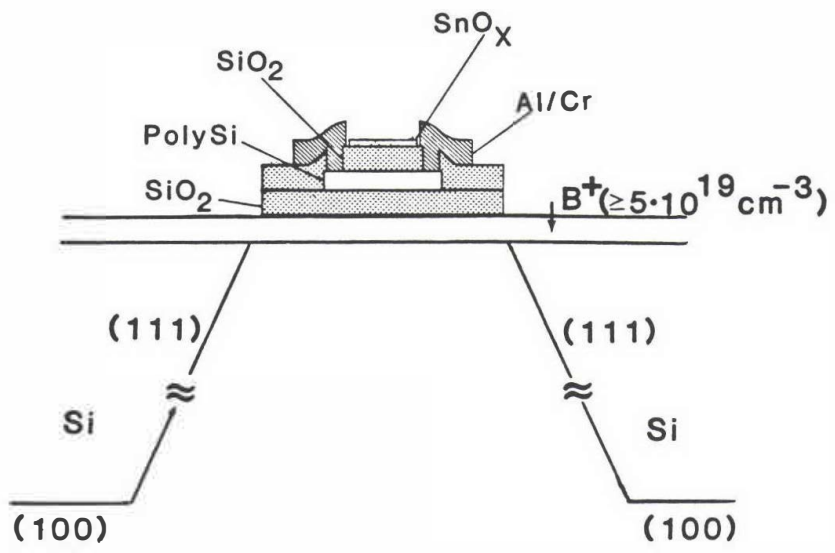

Fig. 2 Schematic diagram of a completed microsensor on a thin $\mathrm{Si}$ membrane. The $\mathrm{Al} / \mathrm{Cr}$ metal contact on tin oxide is not shown.

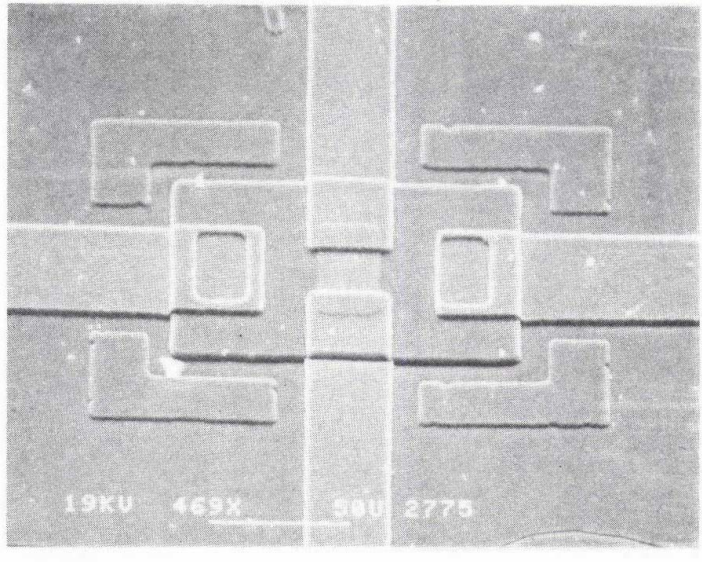

(a)

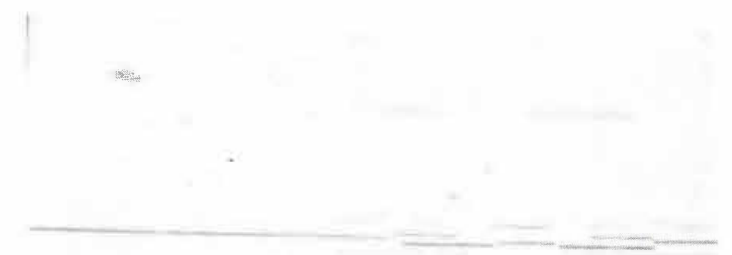

(b)

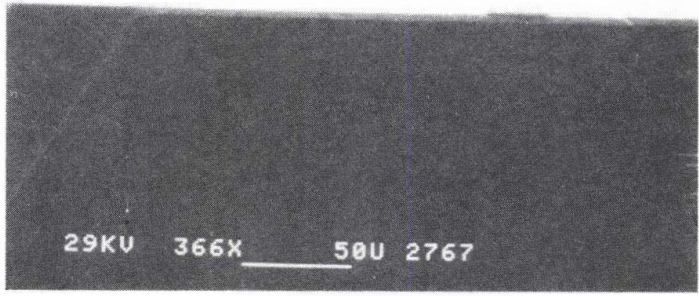
thin Si membrane (a) top view, (b) crosssectional view. 


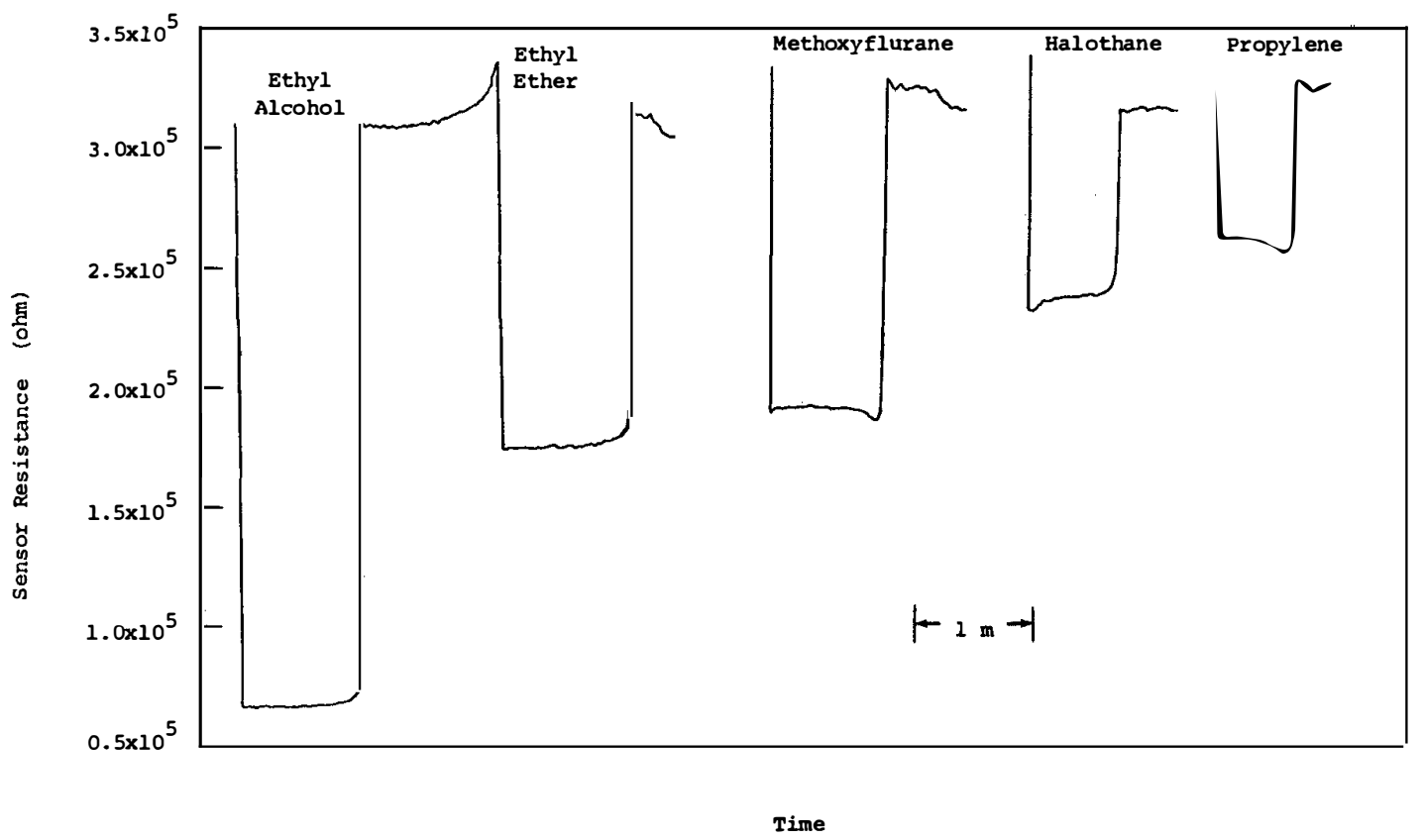

Fig. 4 Sensor responses to $200 \mathrm{ppm}$ of ethyl alcohol, ethyl ether, methoxyflurane, halothane and propylene in air. Sensor material: MOD tin oxide, sensor temperature $=-250^{\circ} \mathrm{C}$.

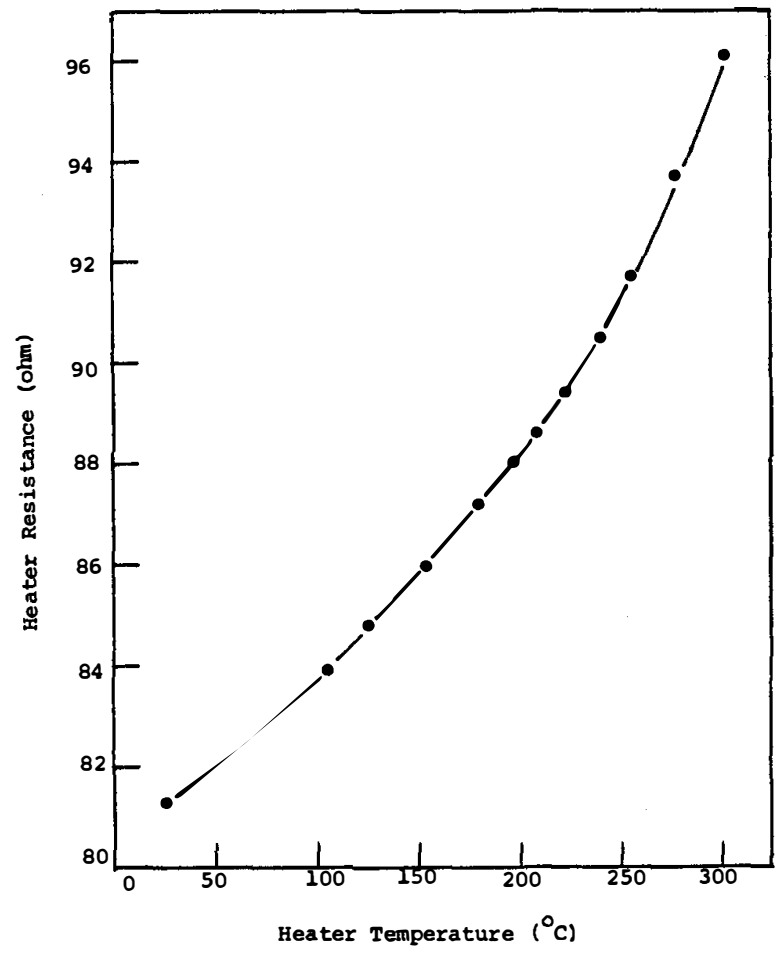

Fig. 5 Polysilicon heater resistance versus temperature. 